\title{
The Dynamical Visual Angle
}

\author{
Ahmad Yousef ${ }^{1}$ \\ ${ }^{1}$ School of Computational Science and Engineering, McMaster University, Hamilton, Ontario, Canada \\ *Correspondence: $\underline{\text { mohamas2@mcmaster.ca }}$
}

\begin{abstract}
This article provides a new insight in the topic of visual angle that had been widely used by vision scientists. The paper disagrees with the current view of the visual angle that considers the pupil as a pinpoint and ignores the dynamics of the pupil and the lens. In another word, this paper presents a new dynamical metric to provide more precision in vision research.

\section{Introduction \& Justification}

Vision scientists, and physiologists had been considering the pupil as a pinpoint when they estimate the visual angle. In practical physics, a pinpoint shouldn't have diameter less than the wavelength of the photon to allow the photon to pass. The human eye can detect wavelengths from 380 to 700 nanometers, and therefore, the minimal pinpoint should be 700 nanometers in diameter to allow the light rays to
\end{abstract} pass. Recent studies, however, shows that light can be squeezed, namely, pinpoints with diameters much smaller than the photons wavelength could allow the light to pass under special concept, but the colors should be similar to the actual colors of the objects. In case of dilated pupil, the eye's opening will have over ten thousand slits; and therefore, the retina will receive over ten thousand projections of the same object. The aforementioned issue should not only complicate the estimation of the visual angle, but also it might threaten our understanding of how human visual system works. This project, however, aims to invade this unsolved issue. Namely, it will start by defining the dynamical visual angle, by deriving its equation from the conventional visual angle equation; which is given by:

$$
\text { Visual Angle }=\tan ^{-1} \frac{x}{d}
$$
where $x$ is the diameter of the object of interest, and $d$ is the distance between the eye and the external object. To develop an equation for the dynamical visual angle, we firstly should imagine what the final accumulative product of the circumstances (see reference 1). Noticeably, it's normal to see dilated pupils in humans with seven millimeters in diameter; that's seven million nanometers which is astonishingly equals to ten thousand times of the smallest acceptable pinpoint. Needless to mention, humans cannot see an object from different angels unless the object has the ability to reflect light rays in omni-directional way. Namely, each point in the object of interest has to reflect light rays, as vectors, in every spatial direction. As we discuss previously, the pupil can be considered as hole that consists of thousands of pinpoints or slits. Each of these slits can create its own projection of the object of interest on the retina, see the central infographic. The lower slit, for example, creates a purple projection of the external object on the retina, and the upper slit creates an orange projection. Needless to mention, those colors are used just to represent the

\section{Retinal Image (Orange Circle) produced by the upper slit} projections produced by ten thousand slits on the retina. It will be literally, a bigger circle that contains ten thousand smaller circles. This is a problematic issue, especially if the object

of interest carries many details on its surface; namely, the details will be overlapped; and therefore, there must be retinal, neurophysiological, or even physical processes to resolve this big issue. Proposed resolutions for this issue will be offered and discussed in the following section. Now, let's derive the equation of the dynamical visual angle that firstly considers only the dynamics of the pupil. It could be written as follows:

$$
\text { Dynamical Visual Angle }=\tan ^{-1} \frac{C * x}{d}
$$

where $C$ is a coefficient reflects the pupil size, and $1 \leq C<$ 2. Importantly, the value of $C$ couldn't exceed 2; geometrical speaking, the projections must intersect. Additionally, dilated pupil is linked to rounded lens (see reference 2, and 3 ). Rounded lens pulls its focal point of the light rays towards the pupil, and therefore, the inverted images 'the projections' on the retina will 
subtend larger degrees of visual angle. Naïve considerations of the dynamics of the lens might generate a generic equation for the dynamical visual angle as follows:

$$
\text { Dynamical Visual Angle }=\tan ^{-1} \frac{m * C * x}{d}
$$

where $m$ is a naïve coefficient that reflects the dynamics of the anatomical lens, and $\frac{1}{C} \leqslant m \leq \mathrm{M}$. Detailly speaking, in case of very constricted pupils, the $m$ parameter might cancel the effect of the dynamical visual angle due to the flattered lens (see reference 2). Namely, the focal point of the light rays is away from the pupil, accordingly the retinal inverted images "projections" will be smaller; and therefore, $m \approx \frac{1}{c}$; however, dilated pupils may maximize the $m$ parameter due to the rounded lens until it reaches to $M$ value. Fine estimation of $M$ value requires special procedures of ophthalmological imaging facilities.

\section{Discussion \& Future Challenges}

The current study raises a significant problem in vision research that should be addressed in several scientific pathways; namely in terms of neurophysiology and/or physics. Neurophysiological speaking, it seems to be very hard to understand what exactly is happening in the retina and in the brain to build a concrete picture of an object instead of multiple overlapped identical ones. Several scenarios, however, can be suggested as follows: 1- The retina might suppress all of the weak projections and allow the strongest one only to signal the brain. 2 - The retina might selectively choose certain projections and forward them to the brain for the purpose of strengthening certain features (depth, and fine details) of the visual awareness of the objects.

3- The retina might send all of the projections to a certain brain region, and that area will simply sum up all of the projections in correlative way.

4- Mixed processes; namely, one or more of the aforementioned scenarios might work collaboratively along with perfect dynamics of ciliary muscle that controls the zonules for the purpose of shaping the lens in different ways to maximize the intersective areas of the inverted projections on the retina. It seems like if all of these operations cannot work perfectly due to the complex situation; the visual awareness might be blurred. It is also very hard to explain how the retina could achieve the center-surround antagonism process unless the first suggestion is the only true suggestion. Otherwise the center-surround antagonism cannot be completely achieved only in the retina (see reference 5).

In terms of physics, there is only one possible scenario that can resolve the 'overlapping' issue. It's through entangled wave functions between the photons and its corresponding photoreceptors. In another word, certain reflected photons of the object (those which might have the best representations of the physical objects) are entangled with certain photoreceptors on the retina where the projection is assumed to reside. The other object's reflected photons will not simply have entangled wave functions with corresponding photoreceptors; and therefore, they will not interact with the retina at all. This scenario might be viewed problematic to a renowned topic in vision research, namely, the visual respective field remapping, see references 6 to 10. In another word, if the photons are entangled with their corresponding photoreceptors, then, the visual stability will remain even under severe saccadic eye movements, and therefore, there will be no need for the theory of receptive field remapping. We here are not trying to violate the remapping theory, we see very disciplined works behind it; however, we think it cannot stand alone in solving the mystery behind the visual stability, namely, we think that the entanglement hypothesis, if true, is an outstanding assistant. Until we reach to a final conclusion about the entanglement hypothesis, we should emphasize that the visual receptive field remapping process should cooperate with the current 'overlapping retinal images' problem to eventually build up a concrete visual awareness. Last but not least, we had already observed encouraging results that might be considered as preliminary supporting evidences for the entanglement hypothesis, see references 11 to 14; accordingly, we think that there might be entanglements doing their best along with receptive field remapping to ultimately stabilize human visual awareness. Essential Notification

Scholars with real time ophthalmological imaging, and eye tracking facilities are welcome to collaborate.

Enquiries should be sent to the author.

Transactional References

[1] Porto etal., (1999). Transmission Resonances on Metallic Gratings with Very Narrow Slits. Physical Review Letters

[2] Yousef, Ahmad. 2019. "Linking Dynamics Between Pupil and Lens." engrXiv. doi:10.31224/osf.io/9c3vk.

[3] Abokyi, etal.,(2017). Caffeine intake is associated with pupil dilation and enhanced accommodation. Eye (Lond) Journal.

[4] P. Cook and J, McReynolds (1998). Lateral inhibition in the inner retina is important for spatial tuning of ganglion cells. Nature Neuro.

[5] Gerald Westheimer, (2004). Center-surround antagonism in spatial vision: Retinal or cortical locus? Vision Research, 2004.

[6] Duhamel, 1992. The updating of the representation of visual space in parietal cortex by intended eye movements. Science Magazine.

[7] Zirnsak, etal., 2014. Visual space is compressed in prefrontal cortex before eye movements. Nature.

[8] Neupane et al. 2016. Dissociation of forward and convergent remapping in primate visual cortex. Current Biology.

[9] Yousef, 2019. "Illusory Snakes Might Be Due to Asynchronized Respective Field Remapping." PsyArXiv. doi:10.31234/osf.io/ve4s8.

[10] C. Paeye; T. Collins; P. Cavanagh (2017). Transsaccadic perceptual fusion. Journal of Vision.

[11] Yousef, 2019. "Voluntary Blinks Stop Binocular Rivalry." PsyArXiv. doi:10.31234/osf.io/z4jrn.

[12] Yousef, 2019. "Motion Triggers Indefinite Stoppage Against Binocular Rivalry." PsyArXiv. doi:10.31234/osf.io/4qbrg.

[13] Yousef, 2020. "Deep Breathing Governs Binocular Rivalry." PsyArXiv. doi:10.31234/osf.io/zr3uk.

[14] Yousef, 2020. "Voluntary Movements Regulate Binocular Rivalry." PsyArXiv. doi:10.31234/osf.io/qk249. 